\title{
Acoustic monitoring of a ball sinking in vibrated granular sediments
}

\author{
Siet van den Wildenberg ${ }^{*}$, Julien Léopoldès, Arnaud Tourin, and Xiaoping Jia ${ }^{\dagger}$ \\ Institut Langevin, ESPCI Paris, PSL Research University, CNRS, 1 rue Jussieu, 75005 Paris, France
}

\begin{abstract}
We develop an ultrasound probing to investigate the dynamics of a high density ball sinking in 3D opaque dense granular suspensions under horizontal weak vibrations. We show that the motion of the ball in these horizontally vibrated glass bead packings saturated by water is consistent with the frictional rheology. The extracted stress-strain relation evidences an evolution of flow behaviour from frictional creep to inertial regimes. Our main finding is that weak external vibration primarily affects the yield stress and controls the depth of sinking via vibration-induced sliding at the grain contact. Also, we observe that the extracted rheological parameters depend on the size of the probing ball, suggesting thus a non-local rheology.
\end{abstract}

\section{Introduction}

A convenient method to determine the viscosity of a simple fluid is to drop a higher density ball in it. The ball accelerates under gravity until reaching a terminal velocity, which is inversely related to the viscosity in the laminar flow regime via the Stokes' law. In a yield stress fluid such as foams, emulsions and granular suspensions, the ball will arrest sinking at a certain depth due to the solid-like friction between the particles. The jamming phase diagram provides a general framework to explain such a transition from a liquid-like flowing state to a solid-like jammed state as a function of the packing density of particles, the confining pressure and the applied shear. However, studies of an intruder sinking in dense granular media are mostly limited to $2 \mathrm{D}$ packings [1] and investigations inside realistic 3D opaque dense granular media [2] remain a conceptual and practical challenge.

Here we report a new experiment investigating the dynamics of a sinking ball (steel) in horizontally vibrated granular sediments, i.e., glass bead packings saturated by water. Using a non-intrusive acoustic probing, we monitor precisely the position $z(t)$ of the steel ball in the 3D opaque granular suspension during sinking. The instantaneous settling velocity $v$ or acceleration $a$ is obtained by numerically differentiating $z$ or $v$ over a time interval. These experimental results are then analyzed with the granular rheology law $\mu(I)$ revealed in dry packings [3], being extended to dense granular suspensions [4-7]. We find that external shaking primarily affects the yield stress [8-10] and consequently controls the depth of sinking. This acoustic monitoring should improve the understanding of the mechanical behavior of dense granular suspensions, quicksands and landsliding.

\section{Experiments}

\subsection{Set-up and procedure}

Our setup consists of a plexiglass container which is coupled via a linker to a shaker and filled with a suspension of glass beads $d \sim 100 \mu \mathrm{m}$ in water. In our experiments, the ball sinking is generated by vibrating the container in the horizontal direction (Fig. 1a). The intruder is a steel ball (we experimented with intruders of different size) which is carefully placed on the surface of the suspension. In the absence of vibrations, the intruder remains on the surface indicating that the granular suspension is in a solid state with a packing density of about $60 \%$.

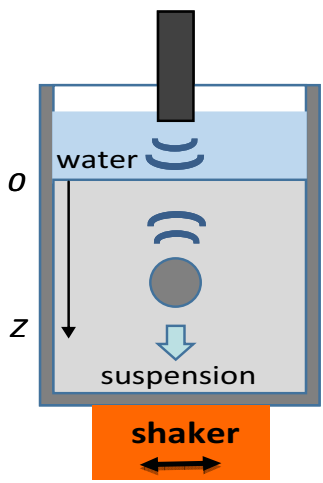

(a)

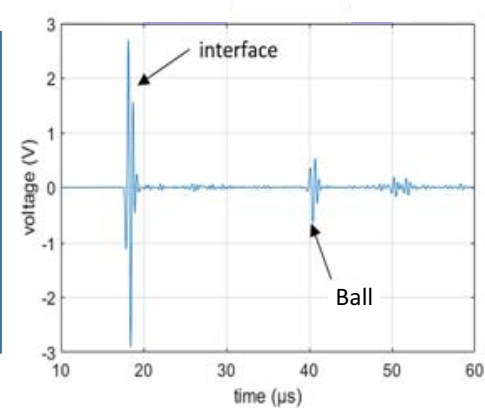

(b)
Fig. 1. (a) Sketch of the experimental set-up. (b) Ultrasonic echos from the interface and the intruder (ball).

\footnotetext{
* Currently at Laboratoire Magmas et Volcans, Université B. Pascal, 63178 Aubière, France

${ }^{\dagger}$ Corresponding author: xiaoping.jia@espci.fr
} 
In contrast, when the suspension is vibrated the intruder starts to slowly sink. To follow the sinking of the intruder in the 3D-opaque suspension we employ acoustic monitoring. For this purpose, a transducer $T$ is placed exactly above the intruder. The transducer is used in reflection mode and it emits short broadband pulses and detects the echos. In this way, a waveform of the echos is acquired at a sampling rate of $\sim 5 \mathrm{~Hz}$. In Fig. $1 \mathrm{~b}$ we show a typical waveform which depicts two echos: one from the interface of the suspension and another one from the intruder. The time of arrival of the echo from the interface remains constant. Instead, the arrival time of the echo from the intruder increases as the distance between the transducer and the sinking intruder increases. From the stack of such waveforms (Fig. 2a), we may determine the position of the intruder $z$ during sinking in the course of time $t$ (Fig. 2b), by multiplying the arrival time of the echo by the sound velocity in water $\left(c_{w}=1500 \mathrm{~m} / \mathrm{s}\right)$. As shown in Fig. $2 \mathrm{~b}$, we observe that the motion of the intruder is slow but continuous and we do not observe short periods of stalling as was reported for sinking in 2D dry granular media [1].

Notice that in certain case we observe not only a small compaction effect at short times but a small dilation of the granular suspension at larger times shown in Fig. $2 b$ (lower trace). For following experiments we have developped a preparation protocol to yields reproducible and stable packings, suppressing this dilation effect.

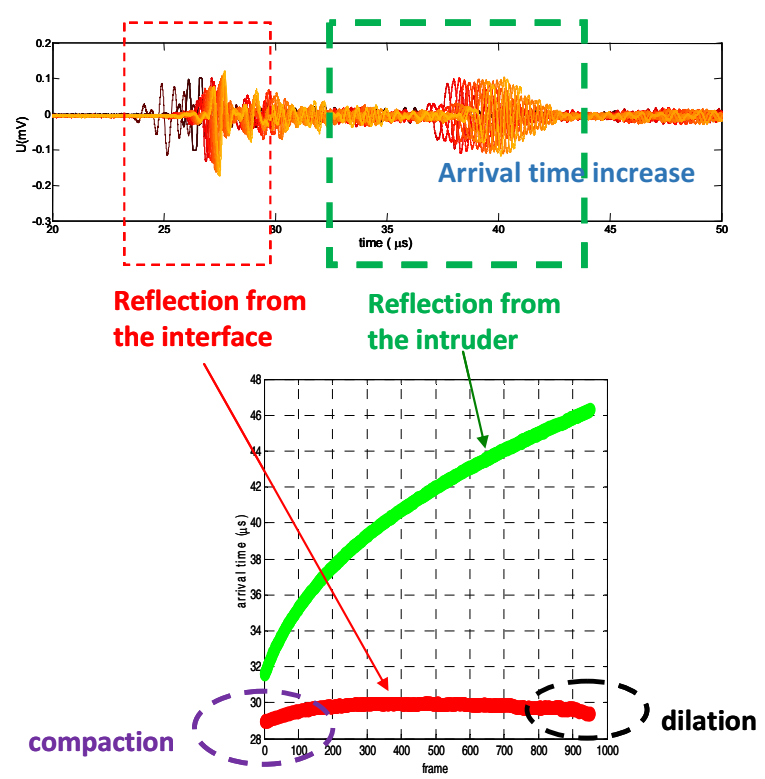

Fig. 2. (a) Stack of waveforms. (b) Position of the intruder during sinking (upper trace) and the position of the suspensionwater interface (lower trace).

\subsection{Results}

To characterize in details the sinking behavior of the intruder, we examine how $z(t)$ depends on the acceleration of vibration $\Gamma$ and the diameter of the intruder $2 R$.
We start by investigating the influence of vibration intensity on the sinking dynamics of the intruder of $R=5$ $\mathrm{mm}$ by varying $\Gamma$ a decade between 0.27 to $2.7 \mathrm{~m} / \mathrm{s}^{2}$ and measuring $z(t)$. Generally, we find that the sinking of the intruder, at each $\Gamma$, can be divided in two regimes (Fig. 3): i) first, a regime in which the intruder decelerates fast (grey parts in the short time), and ii) a quasi steady flow regime in which the intruder moves with an almost constant velocity (in the long time range).

In order to separate these two regimes we calculated, for each $\Gamma$, the instantaneous velocity $d z / d t$ and the acceleration $d^{2} z / d t^{2}$ of the intruder. The $d z / d t$ was obtained, by numerically differentiating $z(t)$ via $[z(t+\Delta t)$ - $z(t)] / \Delta t$, where the time interval $\Delta t$ was set to 20 seconds so to obtain low noise while remaining much shorter than the duration of the data set. The acceleration was calculated from the velocity in a similar way. A cross-plot of the acceleration as a function of the velocity reveals a clear kink separating the fast decelerating and the quasi steady regime (data not shown). We determine the velocity at this kink $(d z / d t)^{*}$ and the associated depth $z^{*}$ which is roughly equal to the diameter of the intruder, suggesting that the fast decelerating regime is likely due to partial submergence of the intruder and boundary effects such as crater formation on the surface just above the intruder. In the following, we will focus on the quasi steady flow regime. For low $\Gamma$ we observe that the intruder decelerates until it finally arrests at a final depth. When increasing $\Gamma$ the intruder reaches larger depths and it keeps decelerating but did not stop completely within our experimental time (Fig. 3).

Next, the influence of the intruder size is investigated by repeating the experiments with intruders of different size $R=4 \mathrm{~mm}$ and $7 \mathrm{~mm}$. The phenomenology of sinking remains similar for all different intruders and we recover the fast decelerating regime and a quasi steady flow regime (data not shown). Interestingly, we find that in the steady flow regime, smaller intruders sink to larger depths; the motion of the intruder appears, therefore, not only to depend on $\Gamma$ but also on its own size $R$.

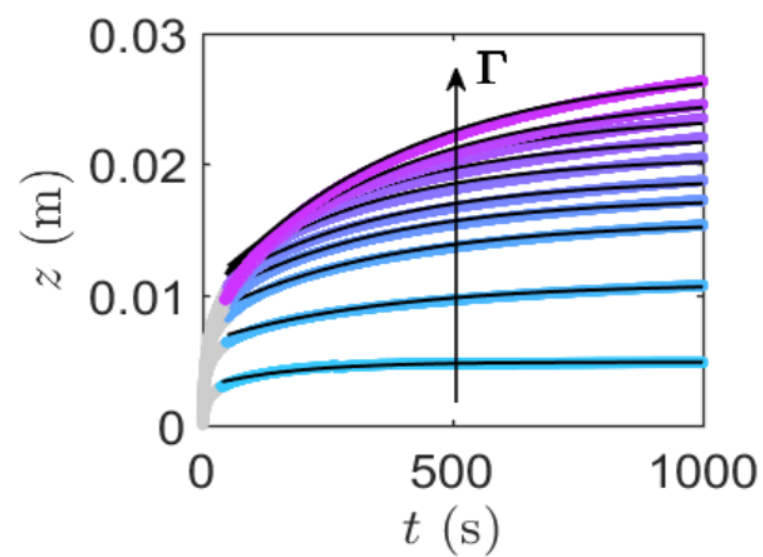

Fig. 3. Dynamics of the ball sinking as a function of time under different vibration intensity $\Gamma$. 


\section{Modelling and discussions}

\subsection{Frictional rheology}

We consider the equation of motion for the intruder sinking along the vertical direction. There are three forces acting on the intruder during its trajectory through the suspension, a force due to gravity, a buoyancy force and a frictional force $F_{s}$ :

$$
m\left(d^{2} z / d t^{2}\right)=(4 / 3) \pi R^{3} \Delta \rho g-F_{s}
$$

where $\Delta \rho=\rho_{s}-\rho_{\text {sus }}$ with the density of the steel ball $\rho_{s}$ $\sim 8000 \mathrm{~kg} / \mathrm{m}^{3}$ and the density of the suspension $\rho_{\text {sus }} \sim$ $1960 \mathrm{~kg} / \mathrm{m}^{3}$.

In order to specify the frictional rheology for our gravitational suspensions, we may estimate the viscous force and inertial forces acting on glass beads saturated by water [4, 5]: $\quad F_{v}=3 \pi \eta_{f} d(d z / d t) \sim 10^{-11} \mathrm{~N}$ and $F_{i}=$ $\rho_{f} \pi d^{2}(d z / d t)^{2} / 6 \sim 10^{-15} \mathrm{~N}$, with the viscosity $\eta_{f}=8.910^{-4}$ Pa.s and the density of water $\rho_{f}=1000 \mathrm{~kg} / \mathrm{m}^{3}$ and the flow velocity $(d z / d t) \sim 10^{-5} \mathrm{~m} / \mathrm{s}$. The Reynolds number $\operatorname{Re}=F_{i} / F_{v} \sim 10^{-4}$ is very small and also the Stokes number $\mathrm{St}=\rho_{p} d^{2} \eta_{f}(d \gamma / d t) \ll 1$ with the density of glass grains $\rho_{p}=2500 \mathrm{~kg} / \mathrm{m}^{3}$ and the strain rate $d \gamma / d t \sim$ $(d z / d t) / R$, indicating that viscous forces dominate at the particle scale and the internal time is given by a viscous scaling. In this case, the constitutive equation for the frictional rheology can be written as $[4,6,7]$ :

$$
\tau=\mu_{0} P+\mu_{1} \eta_{f}(d \gamma / d t)
$$

where $\tau$ is the shear stress, $P=\Delta \rho g z$ the granular pressure due to gravity, $\mu_{0}$ and $\mu_{1}$ are the friction coefficient and viscous parameter, respectively. Eq. 2 shows that for small shear rate, the local stress in a gravitational suspension is a linear combination of a frictional stress and a viscous stress. Consequently, for slow driving the local shear stresse $\tau$ is proportional to $P$, while for fast driving the local shear stresses are proportional to the driving velocity $[7,8]$.

Assuming the frictional force $F_{s}=\tau\left(4 \pi R^{2}\right)$ and considering the quasi-steady regime $d^{2} z / d t^{2} \rightarrow 0$, we derive the corresponding solution of Eq. 1 using the initial condition $z=z^{*}$ at $t=t^{*}$ :

$$
z(t)=\left(A_{0}-z^{*}\right)\left[\left(A_{0} /\left(A_{0}-z^{*}\right)-e^{-k\left(t-t^{*}\right)}\right]\right.
$$

with $A_{0}=\Delta \rho R /\left(3 \mu_{0} \rho_{\text {sus }} g\right)$ and $k=\mu_{0} \rho_{\text {sus }} g /\left(\mu_{1} \eta_{f}\right)$. This equation is used to fit the traces of $z(t)$ in the quasi steady flow regimes with the free fitting parameters $\mu_{0}$ and $\mu_{l}$ (black lines in Fig. 3).

\subsection{Vibration effects on rheological parameters}

The values for $\mu_{0}$ and $\mu_{1}$ obtained from fit are plotted as a function of the vibration acceleration $\Gamma$ in Fig. 4 for different diameters of the sinking ball. We find that for the lowest $\Gamma$ the static coefficient of friction is $\mu_{0} \sim 0.15$, which is slightly lower than the values reported in literature for dense granular suspensions without vibrations $\mu_{0} \sim 0.3$ [6]. For increasing vibration intensity, and, for $\Gamma>2 \mathrm{~m} / \mathrm{s}^{2}$, they approach a constant value.

Moreover, at low $\Gamma$ the values for $\mu_{0}$ and $\mu_{I}$ depend on the size of the sinking ball $R$, suggesting that the local rheology that describes a macroscopic fields trough a constitutive coefficient (Eq. 2) is incomplete and there appear to be other important parameters such as correlation length of force chains in granular packings $[11,12]$.
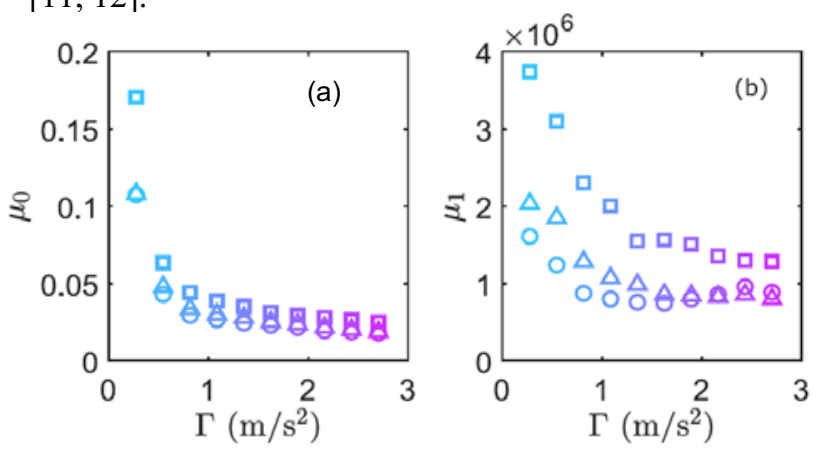

Fig. 4. Rheological parameters versus shaking intensity: (a) static friction coefficient $\mu_{0}$ and (b) viscous coefficient $\mu_{1}$, determined from fitting the $z(t)$ traces like in Fig. 3 by equation (3). The different symbols correspond to intruder size $R=4 \mathrm{~mm}$ (circles), $5 \mathrm{~mm}$ (triangles) and $7 \mathrm{~mm}$ (squares).

To compare our data with rheological measurements performed on the sample scale, we seek to plot the stress versus the strain rate. Specifically, we calculate the shear stress from Eq. 2 using the $\mu_{0}$ and $\mu_{1}$ obtained from the exponential fit and plot the friction coefficient $\mu=\tau / P$ as a function of shear rate $d \gamma / d t$ in Fig. 5. These plots show a plateau at low $d \gamma / d t$ pointing to the friction-like creep regime [13], while for larger shear rate the $\mu$ increases with increasing $d \gamma / d t$ corresponding to the inertial regime. These results are consistent with other rheological measurements in dense granular suspensions [6]. As shown in Figs. 4 and 5, the presence of external vibration strongly affects the yield stress, i.e., the coefficient of friction $\mu_{0}$ as in dry granular packings [8]. Note that for moderate vibration applied here $\Gamma<3 \mathrm{~m} / \mathrm{s}^{2}$, the dilatancy effect caused by vibration would not be significant; the decrease of the yield stress should be primarily due to the reduction of friction coefficient between solid grains caused by acoustic lubrication [10].

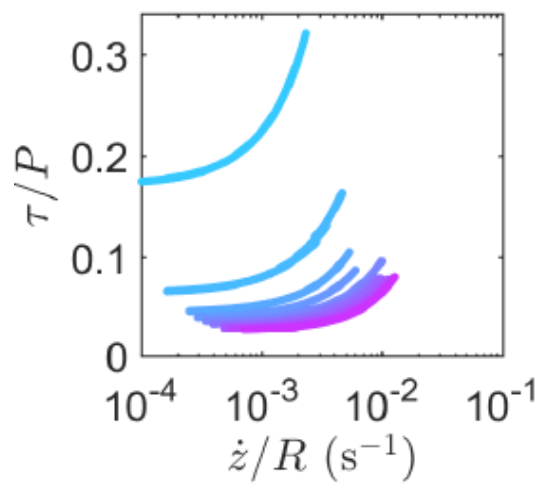

Fig. 5. Effective friction coefficients $\mu=\tau / P$ as a function of normalized sinking velocity (shear rate) of a steel ball $2 R=$ $10 \mathrm{~mm}$, for different vibration intensity $\Gamma$. 


\section{Conclusions}

We developed a novel non-intrusive tool by acoustic probing to follow the dynamics of an intruder emerged in an opaque dense granular suspension. This technique is based on the detection of echos of ultrasonic pulses that propagate through the suspension and are reflected by an intruder. The wavelength $(\sim 0.7 \mathrm{~mm})$ is here much larger than the size of glass particles $(\sim 0.1 \mathrm{~mm})$; nevertheless, we anticipate that this technique can also be adapted to investigate a variety of processes at a shallow depth in more scattering media.

Our main finding is that the sinking of an intruder in a gravitational suspension under vibration crosses over from an inertial viscous regime to a frictional creep regime and this cross-over depends on the vibration intensity. Moreover, the static and dynamic (viscous) friction coefficients decrease with increasing vibration intensity and they increase with increasing intruder size. The dependence of the constitutive friction coefficients on the size of the intruder suggests a non-local rheology.

We thank J.-L. Gennisson and A. Trabattoni for helpful assistance. This work was supported by French LABEX WIFI under references ANR-10-LABX-24 and ANR-10- IDEX0001-02 PSL.

\section{References}

1. R. Harisch, T. Darnige, E. Kolb, E. Clément, Europhys. Lett. 96, 54003 (2011)

2. K. Nichol, A. Zanin, R. Bastien, E. Wandersman, and M. van Hecke, Phys. Rev. Lett. 104, 48 (2010)

3. Da Cruz et al, Phys. Rev. E 72, 021309 (2005); GDR MiDi, Euro. Phys. J.E. 14, 341 (2004)

4. C. Cassar, M. Nicolas, and O. Pouliquen, Phys. Fluids 17, 103301 (2005)

5. S. Courrech du Ponts, P. Gondret, B. Perrin, and M. Rabaud, Phys. Rev. Lett. 90, 044301 (2003)

6. F. Boyer, E. Guazzelli, and O. Pouliquen, Phys. Rev. Lett. 107, 188301 (2011)

7. A. Fall, H. de Cagny, D. Bonn, G. Ovarlez, E. Wandersman et al, J. Rheol. 57, 1237 (2013)

8. J. Dijksman, G. Wortel, L. van Dellen, O. Dauchot, M. van Hecke, Phys. Rev. Lett. 107, 108303 (2011)

9. C. Hanotin, S. Kiesgen de Richter, P. Marchal, L. J. Michot, and C. Baravian, Phys. Rev. Lett. 108, 198301 (2012)

10. J. Léopoldès, G. Conrad, X. Jia, Phys. Rev. Lett. 110, 248301 (2013)

11. K. Kamrin and G. Koval, Phys. Rev. Lett. 108, 178301 (2012)

12. M. Bouzid, A. Izzet, M. Trulsson, E. Clément, Ph. Claudin, B. Andreotti, Eur. Phys. J. E 38, 125 (2015)

13. T. Baumberger and C. Caroli, Adv. Phys. 55, 279 (2006) 